www.jmscr.igmpublication.org

Impact Factor 5.84

Index Copernicus Value: 71.58

ISSN (e)-2347-176x ISSN (p) 2455-0450

crossref DOI: _https://dx.doi.org/10.18535/jmscr/v5i11.121

Journal Of Medical Science And Clinical Research

\title{
Effect of Gender on F Wave Minimal Latency in Healthy Adults
}

\author{
Authors \\ Dr Ahammed Naseem VH ${ }^{1}$, Dr Rajalekshmi G ${ }^{2}$, Dr Zubaida PA ${ }^{3}$, Dr James Jose Jam $^{4}$ \\ ${ }^{1}$ Assistant Professor, Department of Physiology, Government Medical College, Kozhikode, Kerala, India \\ ${ }^{2}$ Professor, Department of Physiology, Government Medical College, Idukki, Kerala, India \\ ${ }^{3}$ Professor, Department of Physiology, Government Medical College, Kozhikode, Kerala, India \\ ${ }^{4}$ Professor, Department of Neurology, Government Medical College, Kozhikode, Kerala, India
}

Corresponding Author

Dr Rajalekshmi G

Professor, Department of Physiology, Government Medical College, Idukki, Kerala, India

\begin{abstract}
Background: $F$ waves are low amplitude late responses due to antidromic activation of motor neurons following peripheral nerve stimulation. It is important in electro diagnosis as it can assess proximal and distal nerve segments unlike conventional nerve conduction studies. $F$ wave minimal latency is the most important and frequently checked $F$ wave parameter. $F$ wave minimal latency is more in males comparing females in many studies. Many authors explain this may be solely due the difference of height between male and female population as height is positively correlated with $F$ wave minimal latency. But some other factors
\end{abstract} unique to males and females can also be reason of this difference.

Aim: Aim of this study was to study the effect of gender in $F$ wave minimal latency independent of height.

Setting and Design: $F$ wave study was done prospectively in 200 carefully screened healthy subjects of age group 20-50 years. The study group included equal proportion of males and females. $F$ wave minimal latencies of median and tibial nerves were found out.

Statistical Analysis Used: All statistical analyses were done with SPSS software version 16. F wave minimal latencies of median and tibial nerves were compared between male and female population using independent $T$ test. Step wise linear regression analysis was done to find out the effect of gender independent of height.

Result and Conclusion: Effect of gender on $F$ wave minimal latency persisted even after nullifying the effect of height. The higher $F$ wave minimal latency in males is not solely due to the difference in height. So gender wise reference data is to be used for proper interpretation of this electro diagnstic parameter

Keywords: $F$ wave study, $F$ wave, Minimal latency, Effect of gender, Nerve conduction.

\section{Introduction}

Electrodiagnostic studies are a physiological extension of the neurological examination ${ }^{1}$. They are used to answer specific questions arising from clinical problems. Clinical electrodiagnosis involves the recording, display and measurement of action potentials arising from central nervous system (evoked potentials), peripheral nerves (nerve conduction studies), and muscles (electromyography) ${ }^{2}$.

Electrodiagnostic procedures are invaluable in the diagnosis of mononeuropathies and to localize and assess the severity of neuropathy. Compression, traction, laceration, thermal, or chemical injury 
may damage one or more components of the peripheral nerves, including the myelin, axons, or supporting nerve structures (endoneurium, epineurium, and perineurium). The pathophysiologic responses to peripheral nerve injuries are demyelination, axon loss, or a combination of both $^{3}$. The most commonly performed tests are motor conduction studies, sensory conduction studies and late responses (F-waves and H-reflex). Electrical stimulation of the nerve initiates an impulse which travels along the motor or sensory nerve fibers. The assessment of conduction characteristics depends on the analysis of compound evoked potentials recorded from the muscle in the study of the motor fibers and from the nerve itself, in the case of the sensory fibers. The same principles apply in all circumstances, although the anatomic course and patterns of innervation dictates the exact technique used for testing a given nerve.

F-waves are low amplitude late responses thought to be due to antidromic activation of motor neurons (anterior horn cells) following peripheral nerve stimulation, which then cause orthodromic impulses to pass back along the involved motor axons $^{2}$. The $\mathrm{F}$ wave has small amplitude, a variable configuration, and a variable latency. Generally Fwave amplitudes are up to $5 \%$ of the orthodromically generated motor response (M response $)^{2}$. Supramaximal stimulus $(25 \%$ above maximal at a rate of not more than $0.5 \mathrm{~Hz}$ ) is required for the elicitation of $\mathrm{F}$ wave.

Since each nerve contains hundreds of motor axons, it is usual to obtain 5-15 F-waves from 20 stimulations. They differ in latency and shape since they normally represent activity from different motor units. The frequency of occurrence is reduced when there is a conduction block anywhere along the nerve. $F$ wave measurements thus reflect conduction along the entire nerve and are therefore particularly useful in the study of general polyneuropathy and also when proximal segments are preferentially involved, as in Guillain-Barré syndrome ${ }^{4}$. In peripheral neuropathies $F$ waves may show clinically significant and measurable changes even before conventional nerve conduction studies are informative ${ }^{5}$.

The minimal latency is most reliable and useful measurement. Study of Weber showed that Fwave minimal latency is the most useful F-wave parameter for the diagnosis of polyneuropathies ${ }^{6}$. It is more frequently and early involved conduction parameter in diabetic subjects ${ }^{7}$. It is the most sensitive measure for detection of nerve pathology and should be considered in electrophysiological studies of diabetic patients ${ }^{8}$.

F wave minimal latency is related to height, limb length and age. So it may vary between different ethnic groups due to the difference in these physiological variables ${ }^{9}$. F wave minimal latency is found to be positively correlated with height ${ }^{9}$. This is due to slower nerve conduction in taller individuals ${ }^{10}$. Many studies showed faster nerve conduction and lower $\mathrm{F}$ wave minimal latency in females comparing male populatio ${ }^{11,12}$. This faster nerve conduction in females along with low $\mathrm{F}$ wave minimal latency sometimes attributed to higher average height of male population. The main aim of this study was to find out the effect of gender independent of height on $\mathrm{F}$ wave minimal latency of the most tested nerves, median nerve in upper limb and tibial nerve in lower limb.

\section{Materials and Methods}

The present study was done in Neurophysiology lab, Government Medical College, Kozhikode to find out the effect of gender on Median nerve $\mathrm{F}$ wave in healthy adults. It was a cross sectional observational study done in normal individuals. The study was done after obtaining approval from the institutional ethics committee. The study was conducted in 200 normal adults (100 males and 100 females) of age 20 to 50 years after informed conscent. Detailed history was taken and clinical examination was done to rule out any systemic or neuromuscular diseases.

Inclusion Criteria: Normal adults of age 20 to 50 years were included in the present study 
Exclusion Criteria: Individuals with systemic or neuromuscular diseases were excluded from the study. Individuals not belonging to Malabar region were also not included in the present study. A RMS EMG EP Mark-II machine was used in the electrophysiology lab. Median and tibial nerves were tested. Subjects were acclimatized to standard room temperature $\left(27^{0} \mathrm{C}+/-2^{0} \mathrm{C}\right)$ for 10 minutes. Supramaximal stimulus $(25 \%$ above maximal) was used. Cathode is placed proximal to anode. The gain was set to 200 microvolt and the sweep speed to 5 milli seconds. At least 10-20 F responses were elicited. F-wave minimal latency was calculated.

Three surface electrodes were used for recording active electrode, reference electrode and ground electrode. The active electrode was placed over the muscle belly. The reference electrode was placed on a nearby tendon or bone away from the muscle. The ground electrode was placed between the stimulator and the active electrode. Grounding is important for obtaining a response that is free of too much artefact. Stimulator consists of two metal pad electrodes placed 1.5 to $3 \mathrm{~cm}$ apart. The Conduction gel should be used to ensure electrical contact.

In median $\mathrm{F}$ wave study active electrode was placed on the centre of Abductor pollicis brevis. Reference electrode was placed at proximal phalanx of thumb 3-4 cm distal to active electrode. Stimulation was given $3 \mathrm{~cm}$ proximal to distal wrist crease between tendons of Flexor carpi radialis and Palmaris longus. In tibial $\mathrm{F}$ wave study active and reference electrodes placed on the centre of Abductor hallucis muscle and over the medial surface of the great toe respectively. Stimulation was done behind and proximal to medial malleolus.

Comparison of $\mathrm{F}$ wave minimal latency between both genders was done by independent $T$ test. Stepwise linear regression analysis was done to find out independent effect of gender. Also height adjusted reference values of $F$ wave minimal latencies were fund.
All statistical analysis was done with SPSS version 16. All relations are significant if $p$ value less than 0.05 .

\section{Observations and Results}

The study population include 100 males and 100 females, who were carefully screened, in the age group 20-50. Detailed history and clinical examinations were done to rule out any systemic or neuromuscular diseases. Average age and height of population was calculated (table 1)

\section{Median nerve}

Mean value of $F$ wave minimal latency of median nerve was found out in both upper limbs separately in males and females. Independent $\mathrm{T}$ test was done to find any significant difference between males and females (Table 2, Figure 1). Results showed a statistically significant high value of $F$ wave minimal latency in male population comparing female population. Linear regression analysis was done to find out the independent effects of height and gender on median F-wave minimal latency (Table 3). Results showed that gender has an independent effect on median F-wave minimal latency, which was more in male independent of height. The result obtained was statistically significant.

\section{Tibial nerve}

Mean value of $\mathrm{F}$ wave minimal latency of tibial nerve was found out in both gender. Independent $\mathrm{T}$ test was done to find any significant difference between males and females (Table 4, Figure 2) which showed a statistically significant high value of tibial $F$ wave minimal latency in male population. Linear regression analysis was done to find out the independent effects of height and gender on tibial $\mathrm{F}$ wave minimal latency (Table 5). Results showed that gender has an independent effect on tibial $F$ wave minimal latency. The result obtained was statistically significant. 
Table 1 Basic information about the study population

\begin{tabular}{|l|c|c|c|c|}
\hline \multirow{2}{*}{ Gender } & \multicolumn{2}{|c|}{$\begin{array}{c}\text { Age } \\
\text { (years) }\end{array}$} & \multicolumn{2}{c|}{$\begin{array}{c}\text { Height } \\
(\mathrm{cm})\end{array}$} \\
\cline { 2 - 5 } & Mean (SD) & Range & Mean (SD) & Range \\
\hline $\begin{array}{l}\text { Male } \\
(\mathrm{n}=100)\end{array}$ & $33.54(9.64)$ & $17-50$ & $170.48(6.74)$ & $150-183$ \\
\hline $\begin{array}{l}\text { Female } \\
(\mathrm{n}=100)\end{array}$ & $35.62(7.82)$ & $18-50$ & $162.3(5.93)$ & $150-178$ \\
\hline
\end{tabular}

Table 2 Comparison of Median F-wave minimal latency of both genders by independent $\mathrm{T}$ test

\begin{tabular}{|l|c|c|c|}
\hline \multirow{2}{*}{ Nerves } & \multicolumn{2}{|c|}{ Mean (SD) } & \multirow{2}{*}{ p value } \\
\cline { 2 - 3 } & Male & Female & $<0.001 *$ \\
\hline Rt. Median & $33.44(0.94)$ & $30.45(0.69)$ & $<0.001 *$ \\
\hline Lt. Median & $33.48(0.91)$ & $30.46(0.7)$ & $<$ \\
\hline
\end{tabular}

Figure 1

* Significant

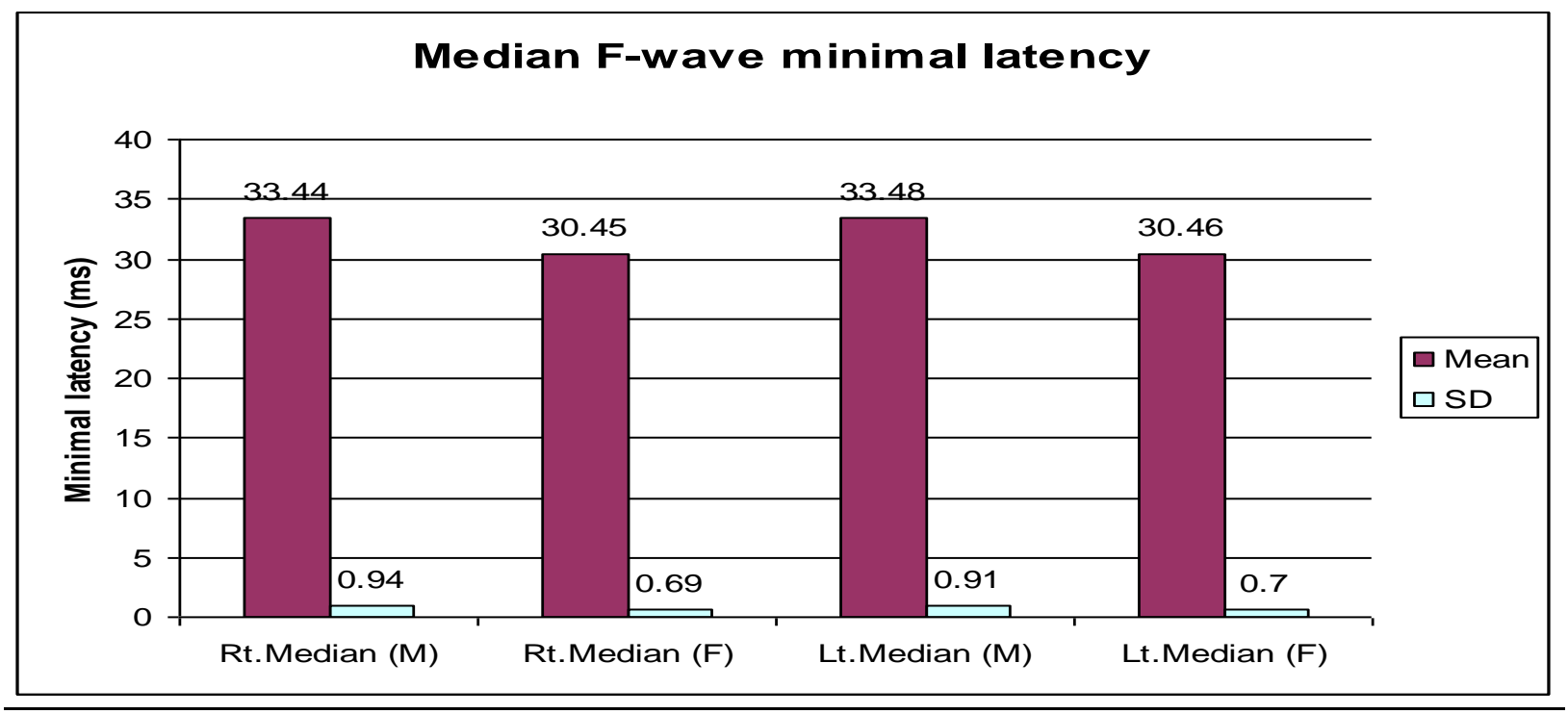

Table 3 Linear regression analysis to find out the independent effect of gender on median F-wave minimal latency

\begin{tabular}{|l|c|c|c|c|c|c|}
\hline \multirow{2}{*}{ Nerves } & \multicolumn{9}{|c|}{ Regression Coefficient } \\
\cline { 2 - 7 } & \multicolumn{2}{|c|}{ Height } & \multicolumn{2}{c|}{ Gender } & \multicolumn{2}{c|}{ Constant } \\
\cline { 2 - 7 } & $\mathrm{B}$ & $\mathrm{p}$ value & $\mathrm{B}$ & $\mathrm{p}$ value & $\mathrm{B}$ & $\mathrm{p}$ value \\
\hline Rt. Median & 0.091 & $<0.001^{*}$ & -2.251 & $<0.001^{*}$ & 20.14 & $<0.001^{*}$ \\
\hline Lt. Median & 0.088 & $<0.001^{*}$ & -2.307 & $<0.001^{*}$ & 20.842 & $<0.001^{*}$ \\
\hline
\end{tabular}

*significant

Table 4 Comparison of tibial F-wave minimal latency of both genders by independent $\mathrm{T}$ test

\begin{tabular}{|l|c|c|c|}
\hline \multirow{2}{*}{ Nerves } & \multicolumn{2}{|c|}{ Mean (SD) } & \multirow{2}{*}{ p value } \\
\cline { 2 - 3 } & Male & Female & $<0.001 *$ \\
\hline Rt. Tibial & $58.11(0.72)$ & $56.4(0.81)$ & $<0.001 *$ \\
\hline Lt. Tibial & $58.07(0.81)$ & $56.39(0.74)$ & $<0$. \\
*significant
\end{tabular}


Figure 2

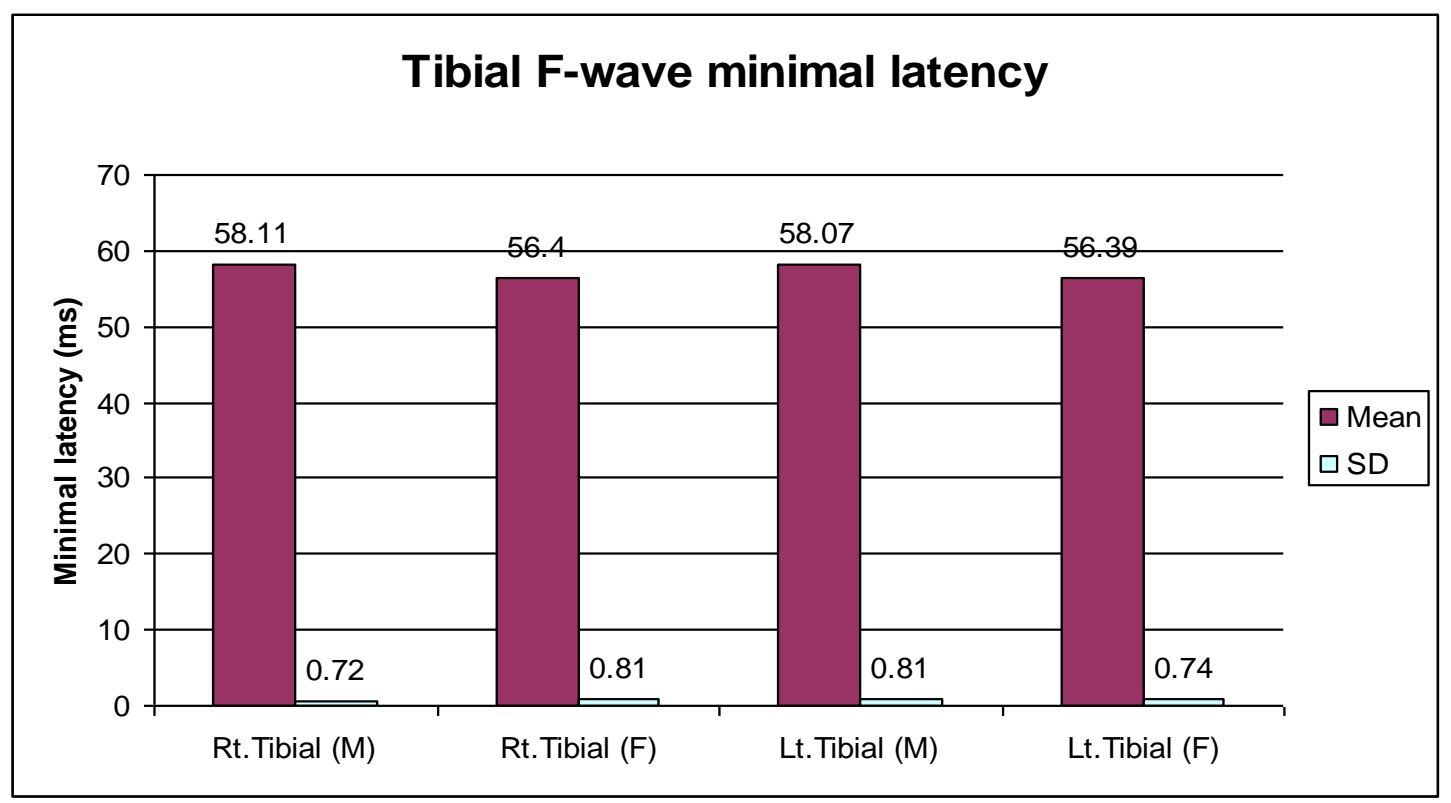

Table 5 Linear regression analysis to find out the independent effect of gender on median F-wave minimal latency

\begin{tabular}{|l|c|c|c|c|c|c|}
\hline \multirow{2}{*}{ Nerves } & \multicolumn{6}{|c|}{ Regression Coefficient } \\
\cline { 2 - 7 } & \multicolumn{2}{|c|}{ Height } & P Value & B & P Value & B Constant \\
\cline { 2 - 7 } & $\mathrm{B}$ & P Value & P Valen \\
\hline Rt. Tibial & 0.078 & $<0.001$ & 1.076 & $<0.001$ & 45.935 & $<0.001$ \\
\hline Lt. Tibial & 0.075 & $<0.001$ & -1.064 & $<0.001$ & 43.375 & $<0.001$ \\
\hline
\end{tabular}

\section{Discussion}

$\mathrm{F}$ wave is a late response recording from a muscle following supramaximal stimulation of its nerve. $\mathrm{F}$ wave response is of variable shape, size and latency. F wave parameters include minimal latency, amplitude, chronodispersion and persistence. Minimal latency is the most frequently used $\mathrm{F}$ wave parameter which is positively correlated with height ${ }^{9}$. The higher $\mathrm{F}$ wave minimal latency in taller individuals indicates slower nerve conduction.

In most of the studies nerve conduction is faster in females $^{13}$. But some investigators attributed this may be due to the other confounding variable, height as the average height will be more in male population ${ }^{14}$. The median and tibial $F$ wave minimal latency was found to be high in male comparing female in the present study. The faster nerve conduction in females obtained in the current study is persisting ever after nullifying the effect of height.
Study conducted by B. Pickett demonstrated that motor conduction velocities of ulnar and peroneal nerves were faster in women independent of any effect of age ${ }^{15}$. It was hypothesised that this difference could be explained if the temperature near nerves was warmer in women or motor axons were larger in females. Study done by James K. Richardson et al showed that the women control subjects had an increased ulnar conduction velocity in the forearm compared to the men ${ }^{11}$. Study done by Nelson et al showed F-wave minimal latency is higher in males than females. Nelson et al also reported F-wave minimal latency of hand muscle to be $31 \mathrm{~ms}$ for females and 34.4 $\mathrm{ms}$ in normal males ${ }^{12}$. The observations from the current study show that, the lower $F$ wave minimal latency in females is not solely due to the difference in height. It may be affected by some other factors also apart from height.

Progesterone may promote myelination by activating the expression of genes coding for 
transcription factors (Krox-20) and/or for myelin proteins (P0, PMP22) ${ }^{16}$. The progesteroneinduced myelination is probably mediated by progesterone receptors, as it is impaired by mifepristone (RU486), a progesterone antagonist. In rodents, the progesterone content of the peripheral nerve is found to be higher in females than in males. The formation of myelin sheaths around axons is a sexually dimorphic process, as the sheaths are thicker in female than in male regenerating nerves ${ }^{17}$. As speed of nerve conduction is directly related to thickness of myelin sheath, this may also contribute the lower value of $F$ wave minimal latency in females compared with males.

\section{Conclusion}

F wave minimal latency is an important electro diagnostic parameter. Unlike the other nerve conduction parameters it helps to diagnosis of both proximal and distal nerve diseases. Like other nerve conduction parameters it varies from population to population. It is affected by physiological variables like height, gender, temperature etc. F wave minimal latency is more in males comparing females. It is positively correlated with height. But the higher $\mathrm{F}$ wave minimal latency in males is not solely due to the difference in height. So a unique gender-wise reference data is needed for each electrophysiology laboratory to find out abnormal cases in its population.

\section{References}

1. Jerry R. Mendell, John T. Kissel, David R. Cornbath: Diagnosis and management of peripheral nerve disorders. Oxford university press; 2001. p. 30-37.

2. Lyn Weiss, Julie K Silver, Jay Weiss. Easy EMG. Philadelphia: Elsevier; 2004. p.17-24, 111-119.

3. Katirji, Bashar. Electromyography in clinical practice: A case study approach. Philadelphia: Mosby Elsevier; 2007. p. 4958.
4. A Mallik, A.I. Weir. Nerve conduction studies: Essentials and pitfalls in practice. J Neurol Neurosurg Psychiatry 2005;76(Suppl II)

5. Zhijun Li, Xiaoqing $\mathrm{Hu}$, Nai Tang. Significance of neuroelectrophysiological tests in the early diagnosis of sub-clinical neuropathay with diabetes mellitus. Journal of Huazhong University of Science and Technology; 2006, Volume 26, Number 4, p. 429-431.

6. Frank Weber. The diagnostic sensitivity of different $F$ wave parameters. J Neurol Neurosurg Psychiatry 1998; 65:535-540.

7. Islam M.R, Bhowmik N.B, Haque A, Haque S, Haque A, Rahman H.R. F wave latency- a frequent and early involved nerve conduction parameter in young diabetic subjects .Mymensingh Med J. Jan 2005; 14(1):46-9.

8. Henning Andersen, Erik Stalberg, Björn Falck. F-wave latency, the most sensitive nerve conduction parameter in patients with diabetes mellitus. Muscle Nerve. 1997 Oct; Volume 20, Issue 10, p. 12961302.

9. Naseem VHA, Rajalekshmi G, Zubaida PA. F wave study in healthy people in Malabar region: Effect of height on $F$ wave minimal latency. J. Evid. Based Med. Healthc. 2016; 3(31), 1449-1453. DOI: $10.18410 / \mathrm{jebmh} / 2016 / 330$.

10. Ahammed Naseem VH, Govindan R, Zubaida PA, Jose J. Normative data of upper limb motor nerve conduction in Northern Kerala population and effect of height on motor nerve conduction velocity. Natl J Physiol Pharm Pharmacol 2016;6:340-344.

11. James K. Richardson, Donald F. Green, Sarah C. Jamieson, F. Clifford Valentin. Gender, body mass and age as risk factors for ulnar mononeuropathy at the elbow. Muscle Nerve. 2001; 24: 551554. 
12. Nelson C, White J.A, Mitchell R.U, Hall C.D. Median nerve $F$ wave conduction in healthy subjects over age Sixty five, Electromyogr Clin Neurophysiol. 1990 Aug-Sep; 30(5): 269-76.

13. Hennessey W.J. Gender and arm length: influence on nerve conduction parameters in the upper limb, Arch Phys Med Rehabil. 1994 Mar;75(3):265-9.

14. Robinson L.R. Influences of height and gender on normal nerve conduction studies. Arch Phys Med Rehabil. Nov1993; 74(11):1134-8 .

15. J. B. Pickett: Motor Conduction Velocity Faster in Women? Diabetologia.1982; 23: 5441

16. Michael Schumacher, Rachida Guennoun, Gilles Mercier, Franck Desarnaud, Pascale Lacor, Jesus Bénavides. Progesterone synthesis and myelin formation in peripheral nerves. Brain Research Reviews. Nov 2001; Volume 37, Issues 13, p. 343-359.

17. H. L. Koenig, W. H. Gong, P. Pelissier. Role of progesterone in peripheral nerve repair. Reviews of Reproduction. 2000; 5, 189-199. 